\title{
Correlation of Structural Modifications by Multiscale Phase Mapping in Filamentary Type HfO2-based RRAM: Towards a Component Specific in situ TEM Investigation
}

\author{
Alexander Zintler ${ }^{1}$, Robert Eilhardt ${ }^{1}$, Stefan Petzold ${ }^{2}$, Nico Kaiser ${ }^{2}$, Sharath Ulhas ${ }^{2}$, Lambert Alff ${ }^{2}$ and \\ Leopoldo Molina-Luna ${ }^{1 *}$ \\ 1. Advanced Electron Microscopy, Materials Science, Technische Universität Darmstadt, Germany. \\ 2. Advanced Thin Film Technology, Materials Science, Technische Universität Darmstadt, Germany. \\ * Corresponding author: molina@aem.tu-darmstadt.de
}

Hafina based resistive random access memory (RRAM), also known as memristors, are promising candidates as next generation non-volatile memory due to their potential for high-density, high-speed, low power consumption and proven compatibility to complementary metal-oxide-semiconductor (CMOS) technology [1]. According to the current understanding, the resistive switching behavior relies on the electric field driven formation and dissolution of oxygen deficient nanoscale conducting paths often discussed as "filaments" [2]. The effects behind the mechanism include the motion of oxygen ions or creation of defects, Joule heating induced by strongly localized currents and interfacial oxygen exchange processes. All these processes are strongly related to the intrinsic material properties which are defined by the local atomic structure.

By using Electron Backscatter Diffraction (EBSD, Fig.1 a-c), XRD pole figure measurements, HR- STEM imaging and automated crystal orientation mapping (ACOM) we were able to analyze the nanoscale grain structure of the multilayer and the arrangement of the monoclinic $\mathrm{HfO}_{2}$ grains in the metal-insulator-metal (MIM) stack. In the present system, the TiN/HfO $/ 2$ Pt stack is deposited on a c-cut $\mathrm{Al}_{2} \mathrm{O}_{3}$ substrate. In an approach to change the macroscopic device properties and achieve a forming free RRAM device, the texture of the $\mathrm{HfO}_{2}$ thin film was controlled by a reactive molecular beam epitaxy synthesis routine. It allows the transfer of the substrate texture to the TiN electrode thin film and finally to the dielectric layer.

Macroscopic datasets show the texture transfer (see XRD pole figures in Fig.1 f-g), where the TiN grains grow with their (111) axis parallel to the surface normal (001) of the c-cut $\mathrm{Al}_{2} \mathrm{O}_{3}$ and the $\mathrm{HfO}_{2}$ layer exhibit (11-1) as their out-of-plane axis. Complementary to the pole figures generated by XRD, the acquisition of high-resolution orientation mappings (Fig.1 e) allow a detailed analysis of the set of existing in-plane rotations for the $\mathrm{m}-\mathrm{HfO}_{2}$ phase. For a single, micrometer sized grain of the TiN bottom electrode a set of three $\mathrm{HfO}_{2}$ grains is observed (Fig. 1 h-i). Indicated by the crystal orientation map, the size of the $\mathrm{HfO}_{2}$ grains is proportional to the film thickness of $10 \mathrm{~nm}$, resulting in grain boundaries that interconnect the top to the bottom electrode as shown in Fig. 2 b. The importance of the grain boundaries mainly arises due to the existence of electronic inter-bandgap states at grain boundaries [4] and the reduced defect formation energies at these sites [3]. These physical properties strongly suggest the initial dielectric breakdown and consequent conducting path formation occurs at the grain boundary. In our electric field dependent in situ TEM studies [5], we demonstrated for the first time how to electrically contact and operate a lamella fabricated in a focused ion beam (FIB). The electrical switching characteristics of the electron-transparent lamella were comparable to a conventional reference device (Fig.2 c) [6]. 
References:

[1] SU Sharath et al., Adv. Funct. Mater. 27 (31) (2017).

[2] R Waser et al., Adv. Mater. 21 (25-26) (2009), p. 2632.

[3] K McKenna and A Shluger, Appl. Phys. Lett. 95 (22) (2009), p. 222111.

[4] K-H Xue et al., Appl. Phys. Lett. 102 (20) (2013), p. 201908.

[5] A Zintler et al., Ultramicroscopy 181 (2017), p. 144.

[6] The authors acknowledge financial support from the Deutsche Forschungsgemeinschaft (DFG) under research grant MO 3010/3-1 and the European Research Council (ERC) "Horizon 2020" Program under Grant No. 805359-FOXON.
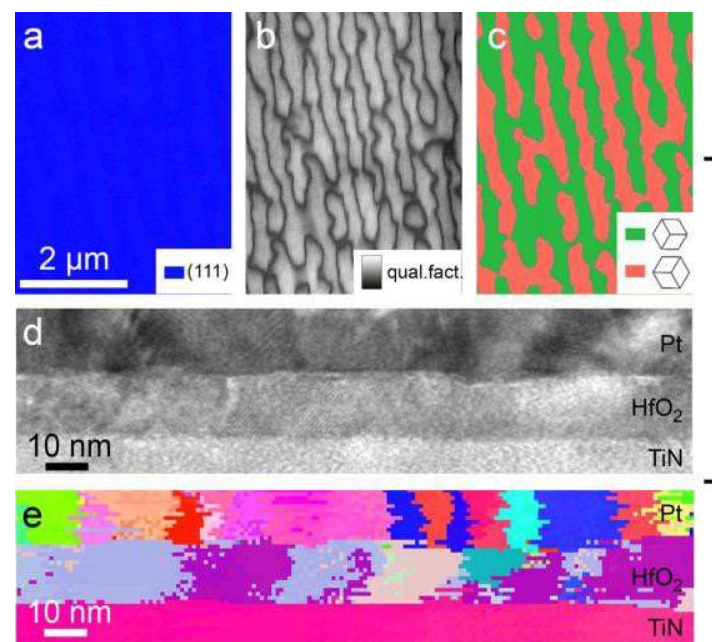

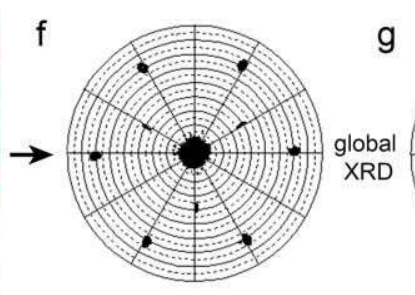

$\operatorname{TiN}(111)$

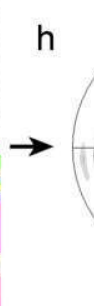

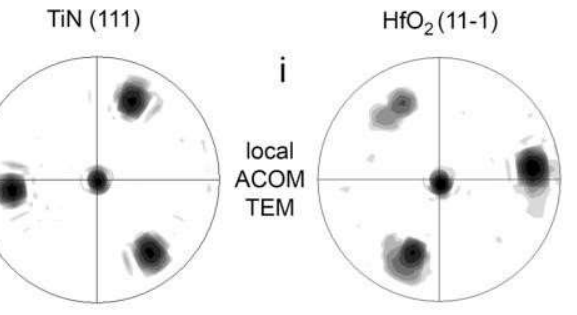

Figure 1. a. Top view TiN electrode inverse pole figure (IPF) map of the (111) oriented TiN layer. b. Image quality (IQ) map of (note boundary regions of quasi-symmetric grains with decreased quality factor). c. Color coded map of the two possible orientations. d. TEM image of the TiN/HfO $/ 2$ Pt MIM stack. e. ACOM phase and orientation mapping of the same area. f-g. XRD pole figures for the TiN (111) and the m-HfO2 (11-1) orientations. h-i. Pole figures derived from the ACOM dataset for the same orientations.
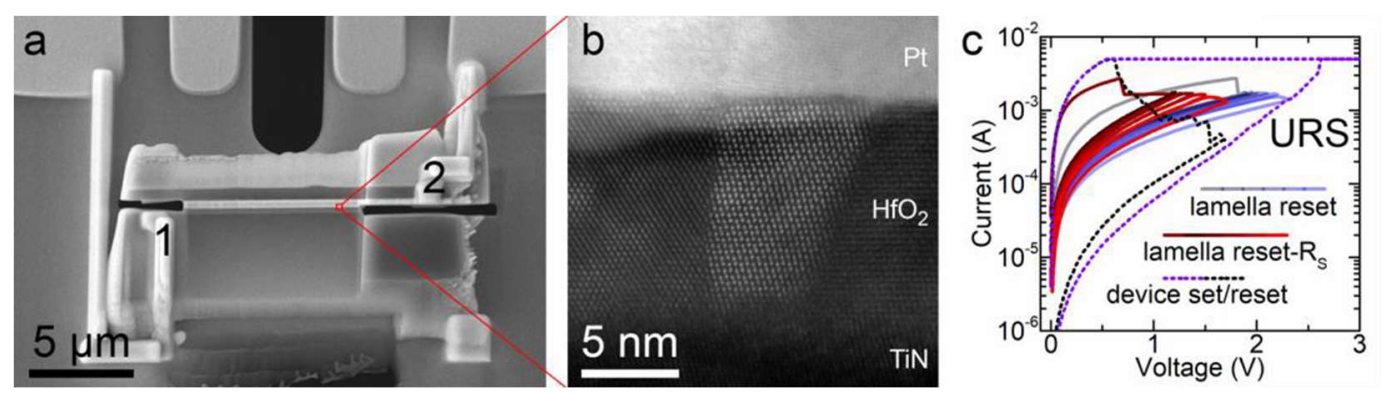

Figure 2. a. SEM image of a FIB prepared $\mathrm{HfO}_{2}$ device on a MEMS based chip used for in situ biasing TEM experiments, contacts for electric field application are denoted as 1 (bottom TiN electrode) and 2 (top Pt electrode). b. Corresponding high resolution HAADF-STEM image of the $\mathrm{HfO}_{2}$ layer and the corresponding electrode interfaces. c. Unipolar resistive switching behavior of a planar device (purple/black dotted curve for set/reset) in comparison to the obtained unipolar reset of the prepared lamella device (blueish curves). 